\title{
ON THE BACTERIOLOGY OF NORMAL ORGANS ${ }^{1}$.
}

\author{
By WILLIAM W. FORD, M.D., D.P.H. \\ Fellow in Pathology, McGill University, Montreal. \\ (From the Molson Pathological Laboratory.)
}

THE question of the presence or absence of micro-organisms in the normal organs of the body has occupied the attention of numerous observers; yet, despite the fact that its solution is not accompanied by any great difficulties, the results of experimentation have been strangely at variance. For instance, Meissner stated that no bacteria capable of development are present in the living tissues of the healthy animal, while Zweifel ${ }^{2}$ (1885) expressed the opposite opinion, namely, that human organs habitually contain germs, that these germs, however, are anaerobic in character, their capability of development being limited by the amount of oxygen present in the blood stream.

Hauser $^{3}$ (1885), who took cultures directly from healthy tissues by the ordinary laboratory methods, and later submitted these tissues to microscopic examination, found almost without exception that they were quite free from bacteria. Welch ${ }^{4}$ in 1891, speaking with especial reference to the Bacillus coli, stated that he had isolated this organism from the internal organs when some distinct lesion of the intestinal mucosa was present, and almost uniformly failed to find it outside the intestinal tract when no demonstrable lesion of the mucosa existed. He stated moreover that the Bacillus coli does not invade the blood and organs in the process of post-mortem decomposition.

1 The following paper is an abstract of a contribution to the meeting of the Association of American Physicians, held in Washington, May, 1900.

2 Zweifel, Ref. Baumgarten's Jahresbericht, 1885, Vol. I. p. 168.

3 Hauser, Ibid., 1885, Vol. I. p. 166.

4 Welch, American Journal of Medical Sciences, 1891, Vol. cr. p. 439. 
Neisser ${ }^{1}$ (1896) and Opitz ${ }^{2}$ (1898), working along quite independent lines, carried out a number of experiments in relation to this question, and reached almost identical conclusions.

Neisser removed the liver, spleen, kidneys, heart, lungs and mesentery from rabbits and guinea-pigs and placed these organs on nutritive media. He observed the usual precautions to avoid contamination. He found that cultures taken at the end of two days were invariably sterile, and concluded therefore that "under normal conditions no bacteria are present in the lymph or blood stream." When on the other hand he first produced artificial lesions of the intestines, and then fed the experimental animals on cultures of various micro-organisms, he found in a considerable portion of cases that he obtained abundant growths from the organs of these animals, either of the bacteria which had been originally administered or of representatives of the normal intestinal flora.

Opitz removed portions of mesenteric glands of cattle killed at the Breslau abattoir. He took them to the Hygienic Institute in that city, where, after carefully sterilising the surface, he excised small pieces and cultivated them on agar and gelatin plates. Finding in the majority of the tissues submitted to this treatment that no bacteria had developed at the end of three days, and that the organisms which were isolated from the small number of tissues which decomposed, were in all cases spore-bearing bacilli (including the Bacillus subtilis, the spores of which are notoriously resistant), Opitz concluded that the mesenteric glands of cattle are normally sterile, that is, that a passage of bacteria through the intestinal wall during digestion does not normally occur.

Neither Neisser nor Opitz record any observations on organs cultivated for a longer period than three days, and in the experiments of the latter, post-mortem changes cannot be positively eliminated, since a considerable time necessarily elapsed between the death of the animals at the abattoir and the taking of the cultures at the Hygienic Institute. To say the least, Opitz was not dealing with absolutely normal structures.

Recent advances in our knowledge of the etiology of chronic fibroid changes in the organs of man, such changes being produced by the long-continued action of bacteria on the tissues, together with the

1 Neisser, Zeitschr. f. Hygiene u. Infektionskr., 1896, Vol. xxıI. p. 12.

¿ Opitz, Ibid., 1898, Vol. xxIx. p. 505. 
special observations of Dr Adami ${ }^{1}$ in Montreal on the presence of minute bodies within the cells of normal and diseased livers, which bodies could only be interpreted on the supposition that they were bacterial in nature, has awakened fresh interest in the question under discussion.

As a consequence a series of experiments has been carried out under Dr Adami's direction, with a view to determining if possible whether more positive results were in any way obtainable.

\section{Methods.}

A number of animals, rabbits, guinea-pigs, dogs and cats, in conditions of fasting and of full digestion, were killed instantaneously by a blow on the back of the neck or by the use of large quantities of chloroform. Immediately after death the skin was removed from the abdomen and a linear incision made through the abdominal wall. The instruments for this purpose were sterilised by boiling and immediately before use were thoroughly heated in the free flame of the Bunsen burner. With fresh instruments, sterilised in the same way, the two kidneys and portions of the liver were excised, and after being cooked in the flame until the surface had been thoroughly scorched to a greyish-brown, they were at once transferred to culturemedia. Three varieties of media were employed. Wide-mouthed bottles containing 50 c.c. of melted gelatin, provided with ground-glass stoppers perforated by small openings plugged with cotton, through which cultures could be taken-served for those organs which were to be preserved at room-temperature. Petri dishes, two inches in depth and two inches in diameter, half filled with neutral agar, were ntilised for the cultivation of organs at the body-temperature. The covers of these Petri dishes were loose and could be lifted from the lower portion sufficiently to allow a culture to be taken from the enclosed organ, without exposing the surface of the agar to the chance of contamination from the air. Finally, large test-tubes containing 50 c.c. of neutral broth and provided with inverted inner tubes similar to those used in the Durham modification of the Smith fermentation tubes, glass rods being passed through the cotton plugs, were employed for "control cultures," corresponding portions of liver and kidney being thus preserved both

1 Adami, British Med. Journ., Oct. 22, 1898. Journ. American Medical Association, Dec. 23, 1899. 
on agar and in broth. Broth tubes were used to determine whether a greater variety of bacteria would develop in fluid than in solid media.

All media were sterilised in the autoclave and at the moment of operation the hot sizzling organs were passed from the flame directly into the agar plates, gelatin bottles or broth tubes, the organs in the latter being thoroughly broken up by the glass rod which was withdrawn through the cotton plug. The organs were then incubated at various temperatures. Cultures were made at the end of the first and third days, by drawing up some of the juice of the organ in a sterile glass pipette, subsequent cultures being made every third day till positive results were obtained or till a sufficient time had elapsed, to demonstrate that the organs were absolutely sterile.

As a later modification of this method, the organs after excision, instead of being passed through the flame, were soaked for an hour in a 1-1000 solution of bichloride of mercury, after which they were washed in sterile water and preserved on sterile plates, either dry, or covered with neutral agar. By this means an insoluble precipitate of albuminate of mercury was formed on the surface of the organ, such a precipitate allowing a complete sterilisation of the surface, without permitting the passage of the sublimate into the interior of the organ and the consequent destruction of any bacteria there present.

\section{Results ${ }^{\mathbf{1}}$.}

From the organs of rabbits preserved in gelatin at a temperature of $22^{\circ} \mathrm{C}$., bacteria were grown in 8 out of 12 cases. These cultures were obtained from the 4 th to the 17 th day. The organisms isolated were Staphylococcus albus, B. coli communis, B. proteus, and several varieties of pathogenic spore-bearing bacilli, the properties of which have been described in another paper.

A number of organs preserved in this way failed to show the presence of bacteria at any time, although cultures were taken at intervals for nearly six weeks.

The cultivation of the rabbit's organs in agar gave results which differed considerably from those obtained with the employment of gelatin. Whatever bacteria were present could develop more rapidly at a temperature of $37^{\circ} \mathrm{C}$, and by the end of the fifth or sixth day

${ }^{1}$ For full tables of the results of these experiments see: Trans. of the Assoc. of American Physicians, Vol. xv. 
many of the organs showed evidences of decomposition, an abundant growth of micro-organisms frequently appearing on the surface of the agar, the bacteria evidently making their way from the enclosed organ through the medium to this point. Cultures were taken from this growth on the agar whenever it appeared, the organ was then removed from the Petri plate, its surface seared with a hot scalpel, and another culture taken from the interior.

The bacteria isolated from the organs preserved in agar were, the Staphylococcus aureus and albus, B. mesentericus vulgatus and fuscus, B. proteus, B. paracolon and some pathogenic spore-bearing bacilli.

The corresponding cultures of the same organs, placed in the broth fermentation-tubes, were identical with the cultures obtained from agar.

In all cases the species of bacteria observed on the surface of the agar plates, were later isolated from the interior of the organ. At the same time, whatever bacteria were obtained from the organs preserved in agar, were grown from the broth tubes as well. The converse of this was not true, however, as in several instances no bacteria were observed in the agar plates while an abundant growth was obtained in the Smith tubes. Evidently the thorough disintegration of the tissues in the fluid medium was more favourable for the development of bacteria, than the preservation of the unbroken organ on a solid substance like agar or gelatin.

Thirty different organs were removed from rabbits and studied in gelatin, agar and broth. Twenty $(66 \%)$ of these organs contained bacteria. The positive results were obtained from the 4th to the 6th day on agar; from the 4th to the 17th day on gelatin; cultures taken on first and third days were sterile.

From the organs of guinea-pigs, 18 in number, cultures were grown in 11 cases $(61 \%)$. The organisms were identical with those seen in the organs of rabbits, and included Staphylococcus albus and aureus, B. mesentericus, B. proteus, B. subtilis and B. coli communis; they appeared in the cultures taken on the seventh day.

The organs of cats furnished most interesting contrasts with the results obtained on the other species of animals. Bacteria were observed in $77.7 \%$ of the samples, 18 organs being studied. These consisted of $B$. mesentericus, B. proteus and B. coli, associated with B. megatherium and B. mycoides.

Besides these, two varieties of bacilli with spores, one a long narrow bacillus, the other a short thick bacillus, were observed in the broth- 
cultures and in smears made directly from the juice of the organs. These bacilli did not grow on any of the usual media although repeated attempts to effect their cultivation were made both aerobically and anaerobically.

In dogs, apparently the same two uncultivatable organisms were observed accompanied by $B$. megatherium, B. mycoides, and rarely by B. zopfi. Of the 18 dogs' organs examined $15(83.3 \%)$ contained bacteria. With both dogs and cats the positive results were obtained on the seventh day.

The sterilisation of the surface of the organs by bichloride of mercury, with which 12 organs from rabbits were treated, did not influence the results to any material extent. . Thus nine $(75 \%)$ of these organs decomposed, the bacteria isolated from them being identical with those obtained with the first set of rabbits : Staphylococcus pyogenes albus, B. mesentericus and B. coli.

The microscopic examination of sections of the organs revealed in many cases well-formed bacilli similar morphologically to those obtained on the culture-media. In other cases only a profusion of coccoid and diplococcoid bodies could be seen, a greater abundance of these bodies being found in the decomposing than in the sterile organs.

The number of these bodies was greater in organs from which $B$. coli was grown, than in those from which spore-bearing bacilli like B. mesentericus or B. subtilis developed.

In the experiments here described, 34 animals were killed, 96 different organs excised and cultivated, and 69 found to contain bacteria. That is, less than $30 \%$ of the organs were sterile. Such results are in more or less direct contradiction to those recorded by other observers, and it thus becomes of interest to seek for some underlying principle which may explain this difference.

The first suspicion to arise in considering our observations, is, that the positive results were due to contamination of the culture-media employed, and that greater attention to the details of asepsis might have insured a larger proportion of negative results.

Putting aside, for the moment, the fact that in every experiment the greatest possible care was taken in the proper sterilisation of media and instruments before operation, while at the operation itself the organs were removed as aseptically as possible; putting aside moreover the fact that control cultures were made in every instance, and that 
these controls remained free from bacteria, considerable interest of a general character attaches itself to the results obtained.

(1) Each species of animal showed its peculiar bacteriology, regardless of the conditions under which the animals were killed or the method in which cultures of the organs were made.

Thus Staphylococcus albus and aureus, B. mesentericus, B. proteus and certain pathogenic spore-bearing bacilli were found in the organs of rabbits and guinea-pigs, while B. mycoides, $B$. megatherium and $B$. zopfii were found in those of dogs and cats, together with two varieties of undetermined bacteria already alluded to.

(2) Each animal, regardless of its species, possessed its distinctive bacteriology. For instance, the same organism was frequently obtained in all the different preparations made from any one particular animal, while in other cases, the entire set of cultures remained sterile.

(3) Each organ preserved its own bacteriology. That is, when any bacteria were isolated from the portions of organs kept in agar, they could always be detected in those portions in the Smith tubes, often, however, associated with other bacteria. Similarly the bacteria found in the growth on the surface of agar were always present in the mixed cultures from the organs.

In short, the cultivation of different bacteria from different species of animals, from different animals and from different organs, regardless of the means employed in the sterilisation of the surface of these organs and regardless of the method of their preservation, demonstrates that these bacteria were present in the organs at the moment of death and were not introduced from without by careless manipulation of instruments or media.

When we compare the methods employed by us with those of others, for instance, Neisser and Opitz, we are struck by the difference in time which was allowed to elapse between the preliminary excision of the organs and the final verdict as to their bacterial condition. With the former observers, all records were taken at the end of two and three days, when the organs were described as sterile. In this work the cultures taken at this time were likewise sterile, thus confirming Neisser and Opitz' results. As these organs were preserved for longer periods however, positive cultures of bacteria were grown in a large proportion of cases, usually on the $6 t h, 7 t h$ or 8 th days, but in some instances as late as the 17 th day. We are thus dealing with a delayed growth of bacteria which is somewhat difficult to explain. Either the bacteria are present in small numbers in the organs 
at the moment of death, and only develop gradually after the organs are excised, or the normal bactericidal or inhibitory substances of the organs, originally powerful enough to prevent the growth of the bacteria are gradually decomposed after the connections between the organs and the animal body are broken, thus permitting of a slow development on the part of the micro-organisms.

In whatever manner these latter questions may be answered, I must maintain, as a result of the experiments here cited, that at least $70 \%$ of the internal organs of our ordinary domesticated animals contain bacteria which are capable of development provided a sufficient time be allowed to elapse between the removal of the organs and their final examination.

I wish to express my thanks to Dr Adami for his constant advice and aid in the conduct of these experiments. 